\title{
Cluster Analysis of Finger-to-nose Test for Spinocerebellar Ataxia Assessment
}

\author{
Michel Velázquez-Mariño ${ }^{1}$, Miguel Atencia ${ }^{2}$, Rodolfo García Bermúdez ${ }^{3,1}$, \\ Daniel Pupo-Ricardo ${ }^{1}$, Roberto Becerra García ${ }^{1}$, Luis Velázquez Pérez ${ }^{4}$, and \\ Francisco Sandoval ${ }^{5}$ \\ 1 Universidad de Holguín, Grupo de Procesamiento de Datos Biomédicos (GPDB), \\ Holguín, Cuba, \\ \{mvelazquez, depupor, idertator\}@facinf .uho.edu.cu, \\ 2 Universidad de Málaga, Campus de Excelencia Internacional Andalucía Tech, \\ Departamento de Matemática Aplicada, Málaga, España, \\ matencia@ctima.uma.es, \\ 3 Universidad Laica Eloy Alfaro de Manabí, Facultad de Ciencias Informáticas, \\ Manta, Ecuador, \\ rodolfo.garcia@live.uleam.edu.ec, \\ 4 Centro para la Investigación y Rehabilitación de Ataxias Hereditarias, Holguín, \\ Cuba, \\ velazq63@gmail. com \\ 5 Universidad de Málaga, Departamento de Tecnología Electrónica, Málaga, España, \\ sandoval@dte.uma.es
}

\begin{abstract}
The Finger-to-nose test (FNT) is an accepted neurological evaluation to study the coordination conditions. In this work, a methodology for the analysis of data from FNT is proposed, aimed at assessing the evolution of the condition of Spinocerebellar Ataxia type 2 (SCA2) patients. First of all, test results obtained from both patients and healthy individuals are processed through principal component analysis in order to reduce data dimensionality. Next, data were grouped in order to determine classes of typical responses. The Mean Shift algorithm was used to perform an unsupervised clustering with no previous assumption on the number of clusters, whereas the $k$-means method provided an independent validation on the optimal cluster number. Experimental results showed the highest internal evaluation for distribution into three clusters, which could be identified as the responses of healthy subjects, SCA2 patients with medium incoordination level, and patients with severe incoordination. A membership function is defined, which allows to establish the subjects' condition based on the classification of their responses. The results support that these protocols and the implemented clustering procedure can be used to accurately evaluate the incoordination stages of healthy subjects and SCA2 patients, thus offering a method to assess the impact of therapies and the progression of incoordination.
\end{abstract}

Keywords: Finger-to-nose test, cluster analysis, incoordination assessment, NeuroScreening Coordination 